\title{
OS CUIDADOS COM A SAÚDE MENTAL DE UMA COMUNIDADE A PARTIR DE UMA PRÁTICA DE ESTÁGIO
}

\author{
Maria Antonieta Araújo Escola Bahiana de Medicina e Saúde Pública
}

\begin{abstract}
Resumo
Este é o relato de uma experiência curricular com graduandos de psicologia ocorrida em um contexto da Atenção Básica à Saúde, no qual atuaram em parceria com Agentes Comunitários de Saúde na identificação e na abordagem em visitas domiciliares aos casos de usuários cadastrados na Unidade de Saúde da Família do Candeal (Salvador$\mathrm{Ba}$ ), portadores de transtorno mental. A prática visou qualificá-los na elaboração e aplicação de roteiros de entrevista e na construção de genogramas, desenvolvendo competências para o contato com pacientes em sofrimento psíquico e com seus familiares. A dinâmica do trabalho levou os alunos à compreensão da rotina de uma Unidade de Saúde da Família, deu apoio as ações dos agentes comunitários de saúde junto à famílias identificadas, construiu uma ferramentas de acompanhamento familiar, contribuindo com o trabalho dos profissionais de saúde da USF e valorizou as ações da psicologia comunitária no âmbito da atenção básica à saúde. Os resultados dessa atividade prática foram compilados e os dados coletados nas entrevistas feitas pelos estudantes foram tratados estatisticamente e apresentados em gráficos e tabelas aqui descritos, os quais foram apresentados às equipes de saúde.
\end{abstract}

Palavras Chave: Psicologia; Comunidade; Formação; Extensão; Saúde da Família.

\section{THE CARE OF A COMMUNITY MENTAL HEALTH FROM A COURSE OF TRAINING}

\begin{abstract}
This is the report of an curriculum experience with undergraduate students from psychology occurred in a context of Primary Health Care, which worked in partnership with community health workers in identifying and addressing on home visits to the cases of registered users at the Family Health Unit of Candeal (Salvador-Ba) who have mental disorders. The practice aimed to qualify them in developing and implementing interview scripts and constructing genograms, developing skills for contact with patients in psychological distress and with their families. The dynamics of the work led the students to understand the routine of a Family Health Unit, it has also supported the actions of community health workers next to identified families, it built family monitoring tools, contributing to the work of health professionals of USF, and it appreciated the actions of community psychology in the context of primary health care. The results of this practical activity were compiled and the data collected in interviews conducted by students were statistically analyzed and presented in graphs and tables herein, which were presented to teams of health.
\end{abstract}

Keywords: Psychology; Community; Education; Extension; Family Health. 


\section{NOTAS INTRODUTÓRIAS}

Este é o relato de uma experiência curricular com graduandos de psicologia ocorrida em um contexto da Atenção Básica à Saúde, no qual atuaram em parceria com Agentes Comunitários de Saúde na identificação e na abordagem em visitas domiciliares a usuários da Unidade de Saúde da Família do Candeal (Salvador-Ba).

A experiência apresentada refere-se a uma prática de estágio básico curricular do $3^{\circ}$ semestre do curso de Psicologia da Escola Bahiana de Medicina e Saúde Pública (EBMSP), integrando dois componentes curriculares do referido semestre - Estágio Básico e Técnicas de Investigação em Psicologia.

A prática ocorreu no âmbito da Atenção Primária e, portanto, no nível dos cuidados básicos com a saúde, tendo como contexto uma Unidade de Saúde da Família (USF) em um distrito sanitário de Salvador, onde uma turma de vinte e um graduandos em psicologia atuou em parceria com duas equipes da Estratégia de Saúde da Família, que, na Unidade de Saúde da Família (USF) de referência, compõem um efetivo dos seguintes profissionais: duas médicas, duas enfermeiras, uma dentista, quatro técnicas de enfermagem, uma técnica de saúde bucal e oito agentes comunitários de saúde.

Os antecedentes da prática efetivada estavam alicerçados em algumas constatações de docentes e discentes que haviam realizado ou estavam realizando atividades de extensão na referida unidade de saúde, desde 2006. Entre elas destacavam-se: (a) a dificuldade das equipes de saúde lá atuantes, na abordagem e encaminhamento de casos de usuários cadastrados naquela USF cujos agravos à saúde apresentados estavam circunscritos, prioritariamente, no nível da saúde mental, (b) a demanda crescente da comunidade pelo serviço de atendimento psicológico lá implantado pelo curso de Psicologia da EBMSP como atividade de extensão, (c) a sensibilidade e disponibilidade da equipe médica para a escuta do sofrimento psíquico dos usuários que a ela demandavam e da necessidade que tinham de mais informações sobre o funcionamento da rede de apoio especializada para os cuidados com as questões psicológicas e psiquiátricas para fins de encaminhamento e orientação. 
Tais constatações foram responsáveis pela elaboração de algumas estratégias possíveis de serem implementadas devido à parceria interinstitucional que viessem a favorecer os cuidados no âmbito da saúde mental a partir atenção primária à saúde integral na unidade citada.

A primeira delas foi a mobilização de alguns docentes do curso de Psicologia que tinham aproximação com o contexto em discussão em torno da elaboração de um projeto de especialização cujo tema, focando-se nos cuidados com a saúde mental a partir da atenção básica, pudesse ser oferecido não apenas aos profissionais parceiros mas também a todos aqueles interessados e atuantes no nível da atenção primária da rede do Sistema Único de Saúde (SUS).

Em paralelo, foi construído um plano de intervenção de apoio psicossocial, integrado à rotina de trabalho das equipes da USF, possível de ser executado por graduandos em Psicologia do $3^{\circ}$ semestre, como a prática do Estágio Básico que deveria ser cumprida naquele semestre letivo.

Em uma linha pedagógica interdisciplinar, o plano de estágio pode ser compartilhado por outro componente curricular já citado anteriormente - Técnicas de Investigação em Psicologia cujo conteúdo incluía o aprendizado dos alunos na elaboração e aplicação de roteiros de entrevista e a construção de genogramas, um instrumento gráfico que "possibilita a visualização de grande número de dados sobre determinada família, incluindo seu passado hereditário com os riscos que oferece aos membros atuais, juntamente com influências clínicas, sociais e interacionais". (1, p. 1)

Dessa forma, foi estruturada uma abordagem prática de apoio às duas equipes de saúde atuantes naquele contexto da Estratégia de Saúde da Família, a qual será aqui sistematizada. Tal sistematização detalhará o delineamento do trabalho realizado, seu plano de ação, fazendo uma discussão teórica sobre as competências e, finalmente, seus resultados, como apresentados a seguir.

\section{SOBRE O DELINEAMENTO DO TRABALHO}

O plano elaborado para o estágio, além de ter suas diretrizes incorporadas à rotina dos profissionais da unidade, buscando não interferir na organização do trabalho lá realizado, adequou-se à infraestrutura da USF e criou um espaço de integração dos acadêmicos com os 
ACS, principais responsável pelas visitas domiciliares e contato com os núcleos familiares, importantes pré-requisitos para a ação a ser implementada.

Tal ação concentrava-se no mapeamento pelos alunos, de núcleos familiares nos quais os ACS já haviam identificado previamente casos de cadastrados com demandas mais emergentes no campo da saúde mental.

Cabe aqui uma observação: o papel profissional do agente comunitário de saúde, dentre os outros que atuam na Estratégia de Saúde da Família (ESF), traduz-se como um lugar de destaque nas articulações necessárias aos cuidados com a saúde da comunidade.

Na pesquisa de Silva e Dalmaso, ${ }^{(2)}$ são identificadas duas importantes dimensões desse papel no que diz respeito à sua atuação, a partir das propostas da ESF: uma dimensão técnica, relacionada ao atendimento aos cadastrados nas USFs com intervenções para a prevenção de agravos e monitoramento de questões específicas identificadas pelas equipes de saúde, devendo, para isso, realizar visitas domiciliares sistemáticas, e uma dimensão de viés político no sentido de organização da comunidade, de transformação dessas condições e não apenas de solidariedade quando da inserção da saúde no contexto geral da vida da população por eles assistida.

Para que essas dimensões se cumpram é necessário, contudo, uma qualificação não apenas técnica, mas, um entendimento da perspectiva política e ideológica da ESF por parte daqueles profissionais e também de todos os que compõem uma equipe atuante em uma USF.

No estágio em questão, as ações da comunidade foram precedidas por encontros de integração dos acadêmicos com os ACS, quando todos puderam discutir sobre suas expectativas e representações simbólicas sobre a saúde integral, a saúde mental, o sofrimento psíquico e compartilhar o conhecimento prévio que tinham sobre transtorno mental. Os ACS puderam também relatar os desafios que cercam o seu trabalho cotidiano na comunidade na perspectiva do tema discutido.

Buscamos com isso, uma maior interação inicial entre os atores, levando-os a um entendimento da contribuição de todos em um processo que poderia ser considerado um piloto em se tratando de uma intervenção acadêmica extramuros, adequando a aprendizagem à realidade empírica. 
Um levantamento entre os estudantes sobre essa fase inicial do estágio levou à coleta de alguns dados significativos, em questionário aplicado para avaliação do processo. Segue uma amostra de respostas encontradas.

O primeiro contato me causou muita expectativa e vontade de dar o máximo de mim enquanto estudante, em busca de novos conhecimentos. (Aluno 1)

Foi de forma bem esclarecedora no que diz respeito à apresentação do planejamento e as experiências de médicos e agentes comunitários. (Aluno 5)

Foi diferente e inesperado, muito proveitosa a experiência, pois a conversa e a interação dos profissionais com os estudantes é extremamente necessária. (Aluno 3)

Foi de grande importância conhecer os agentes comunitários, compreendendo as suas preocupações, medos e expectativas. (Aluno 19)

O planejamento do trabalho, que teve a duração de um semestre letivo, seguiu um roteiro de acordo com o que será apresentado a seguir, em um diálogo com o campo das habilidades e competências.

\section{SOBRE O PLANO DE AÇÃO: HABILIDADES E COMPETÊNCIAS}

Passada a fase inicial de integração dos estudantes com os profissionais da USF, como explanado no item anterior, os primeiros foram divididos em dois subgrupos de dez e onze alunos respectivamente, compondo, cada um deles, uma equipe com mais quatro agentes comunitários, a fim de que fosse realizado o trabalho.

Procedeu-se então à identificação de núcleos familiares cadastrados naquela unidade que tinham entre seus pares, algum(uns) de seu(s) membro(s) com diagnóstico no campo dos transtornos mentais ou que apresentava(m) sintoma(s) de sofrimento psíquico necessários de serem vistos de forma mais apropriada pela sua rede social mais próxima e/ou pela rede de cuidados com a saúde mental do território.

Inicialmente, cada agente elegeu uma média de três casos a serem abordados pela ação dos estagiários. Eles lhes foram descritos e tiveram seus aspectos contextuais discutidos, a fim de que se procedesse ao estudo, nos encontros de supervisão junto às docentes dos componentes 
curriculares (Estágio Básico e Técnicas de Investigação em Psicologia I). Nesses encontros foram estruturadas as abordagem possíveis, tendo em vista a singularidade de cada família e de cada sujeito da ação pretendida. Dessa forma, cada estagiário comprometeu-se com uma família e tinha um ACS como referência.

A partir daí, a organização do estágio se deu em diferentes etapas as quais incluem: pesquisa dos prontuários, visitas domiciliares com os agentes, aplicação de roteiros de entrevista e do genograma - instrumentos que contaram com a orientação da professora de Técnicas de Investigação em Psicologia I - reuniões com as equipes de saúde e as supervisões sistemáticas com o docente responsável pelo estágio.

Com uma carga horária de 60 horas, essa atividade curricular teve como objetivo geral fortalecer a abordagem à saúde mental nos cuidados com a saúde integral de usuários de uma Unidade de Saúde da Família. Os objetivos específicos foram expressos como:

a) levar os alunos à compreensão da rotina de uma Unidade de Saúde da Família;

b) ampliar a rede de apoio aos casos de saúde mental na comunidade a partir de uma ação de trabalho integrada à rotina das ações dos agentes comunitários de saúde;

c) construir ferramentas de acompanhamento familiar, contribuindo com o trabalho dos profissionais de saúde da USF;

d) valorizar as ações no campo da psicologia comunitária no âmbito da atenção básica à saúde.

Avançando na análise desses objetivos, é pertinente um diálogo com o campo que na atualidade tem trazido acréscimos ao processo pedagógico - o que trata da construção de habilidades e competências.

Para efeito de contextualização, vale ressaltar que historicamente tais conceitos têm sido estudados, tanto pelo campo do trabalho, como pelo campo da educação.

No primeiro caso os estudos científicos foram iniciados com as pesquisas de Zarifian, ${ }^{(3)}$ sociólogo francês que assim definiu competência:

A competência é o "tomar iniciativa", é o "assumir responsabilidade" do indivíduo diante de situações profissionais com as quais se depara [...]. Essa formulação enfatiza o 
que muda fundamentalmente na organização do trabalho: o recuo da prescrição, a abertura de espaço para a autonomia e a automobilização [sic] do indivíduo. Cada uma dessas palavras tem importância em si mesmas[...]..$^{(3, \text { p. } 68)}$

Buscar legitimar a concepção de competências para o espaço laboral, significou acompanhar a evolução do conceito de trabalho com suas novas exigências em direção à flexibilização, exigindo com isso trabalhadores criativos e empreendedores.

No campo da educação, sobretudo da superior, competência ganha um significado a partir do Projeto Tuning para a América Latina ${ }^{(4)}$ A propósito, a palavra tuning é derivada do termo inglês tune, que significa afinar. Nascido no contexto daquele nível da educação, o projeto reuniu primeiramente 156 universidades europeias. Em seguida foi ampliado para a América Latina com a meta de identificar e intercambiar informações e melhorar a colaboração entre as instituições de educação superior para o desenvolvimento da qualidade, efetividade e transparência. Para isso, foi tomada como proposta inicial, a busca de pontos comuns de referência, centrados nas competências.

Uma análise de Andrade Cázares ${ }^{(5)}$ sobre o documento que consolida o referido projeto afirma que o conceito de competência em educação se apresenta como uma rede conceitual ampla que faz referência a uma formação integral do cidadão, por meio de novos enfoques, destacando a aprendizagem significativa em diversas áreas, tais como: cognitiva (saber), psicomotora (saber fazer), afetiva (saber ser - atitudes e valores). Isso abarca todo um conjunto de capacidades que se desenvolvem, segundo o autor, através de processos que conduzem a pessoa responsável, a ser competente para realizar múltiplas ações nos âmbitos social; cognitivo; cultural; afetivo; laboral; produtivo, onde teria oportunidade de evidenciar sua capacidade de resolver um problema dado, dentro de um contexto específico e dinâmico.

Isso afina-se com a definição de competência proposta por Perrenoud, ${ }^{(6)}$ para quem ser competente é ter a capacidade de articular um conjunto de esquemas, permitindo a mobilização de conhecimentos adquiridos em uma dada situação de maneira correta e com discernimento.

Em que pese a fluidez ou a "flutuação semântica" do termo competência, Segnini (7) observa, existir um consenso entre os pesquisadores de que este conceito não expressa "[...] apenas um conteúdo cognitivo que exprime uma relação entre o sujeito e as situações concretas 
sobre as quais o espera" ${ }^{(7,}$ p12). Essa dimensão cognitiva não seria a única a ser mobilizada na competência. Se o fosse, estar-se-ia tratando apenas da qualificação técnica para o trabalho.

Segnini ainda cita Tanguy ${ }^{(7, p .2)}$ que considera competência como a "[...] idéia-chave do mundo contemporâneo à medida que logra dois universos paralelos, tornando-os contíguos": o do trabalho e o da educação

A noção de competência dá conta da passagem dos saberes institucionais disciplinares, no plano da educação, para o acento no uso mais livre e criativo das faculdades cognitivas do sujeito e, no mundo do trabalho, dos requisitos exigidos pelo posto de trabalho à capacidade de lidar com situações não propriamente descritas por ele. ${ }^{(7, \text { p12) }}$

Seguindo uma concepção pedagógica focada na construção de competências, o espaço de práticas elaborado para essa atividade buscou levar o aluno a ser capaz de:

a) Identificar aspectos da realidade social, econômica e cultural que interferem na produção de qualidade de vida e saúde dos indivíduos e grupos familiares abordados.

b) Articular contextos e processos psicossociais na interpretação de comportamentos individuais e grupais em saúde.

c) Construir e aplicar instrumentos para obtenção de informações psicossociais e institucionais no campo da saúde.

d) Conduzir as relações profissionais e acadêmicas com colegas e usuários dos serviços prestados, a partir dos princípios éticos que regem a profissão.

e) Conhecer conceitos básicos do campo da saúde mental.

O plano de ação definido para o alcance de tais competências no estágio em discussão reproduziu o que Burnier ${ }^{(8)}$ concebeu como características determinantes para um processo formativo dessa natureza. Para essa autora, tais processos devem ser um lugar de participação consciente e crítica e de colaboração ativa, sendo eles próprios avaliados coletiva e permanentemente se o que pretendemos é formar trabalhadores-cidadãos críticos, criativos e autônomos. Ela sugere que nós, educadores, devemos estar atentos para a objetividade e clareza na nossa comunicação com os alunos, deixando muito bem explicitada cada etapa do processo educativo, de forma que compreendam amplamente o seu valor. 
Na prática em questão, os encontros de supervisão que tínhamos com os dois subgrupos de estudantes na unidade de saúde, cumpriam essa função. A cada semana estruturava-se o passo seguinte e avaliava-se coletivamente o que houvera sido realizado. Mensalmente, havia uma reunião com todos os profissionais da unidade, quando eram socializados os casos e pensava-se nas estratégias de intervenção devidas que foram de natureza diversa: (a) encaminhamento do caso em estudo ou de algum familiar para o atendimento psicológico da unidade onde estagiários do $10^{\circ}$ semestre realizavam atendimento clínicos (b) articulação com o CAPS de referência no território para visita domiciliar em alguns casos de alta complexidade, envolvendo quadros de transtorno mental (c) atenção psicossocial atrelada ao trabalho educativo de orientação sobre o acesso à rede pública de serviços quando se fez necessário (d) encaminhamento de alguns sujeitos ou familiar para os grupos de educação em saúde que se realizam na comunidade mediante uma atividade de extensão da EBMSP. ${ }^{1}$

A partir do exposto será feita a seguir uma apreciação dos resultados da prática de estágio na comunidade.

\section{SOBRE OS RESULTADOS DA ATIVIDADE DE ESTÁGIO REFERENTES À SAÚDE MENTAL}

Promovendo o enriquecimento do trabalho e organizando-o de maneira a dar um feedback às equipes de saúde da USF, os estagiários fizeram um "mutirão" realizando o tratamento dos dados coletados nos roteiros de entrevista, sendo estes compilados ${ }^{2}$ e apresentados os resultados em uma reunião de encerramento do semestre letivo.

No campo da prática, a ação favoreceu aos profissionais das equipes de saúde responsáveis por aqueles cadastrados, um maior conhecimento da história dos sintomas que eles apresentavam, fossem de ordem psicológica ou psiquiátrica, além da valorização dos aspectos psicossociais da vida desses sujeitos. Puderam também estar inteirados do plano de tratamento aos quais já haviam sido submetidos aqueles que já eram ou seriam seus pacientes, uma vez que

\footnotetext{
${ }^{1}$ A citada atividade trata-se do Programa Candeal, o qual se constitui por grupos temáticos de educação em saúde que ocorrem regularmente em diferentes locais da comunidade e são coordenados por equipes de estudantes e professores dos diversos cursos da EBMSP. De natureza interdisciplinar, o trabalho aborda as diversas dimensões da saúde nos trabalhos grupais.

${ }^{2}$ Mascarenhas P. Protocolo de acompanhamento em saúde mental no PSF: estágio na Unidade de Saúde da Família. Salvador: EBMSP; 2010. Apresentação PowerPoint.
} 
habitavam na área circunscrita à USF, direcionando, com isso, a uma melhor conduta no seu tratamento quando das intervenções necessárias naquela unidade de saúde.

A ação descrita pode ser considerada como um ensaio de contribuições possíveis de trabalho associando o serviço público e o espaço acadêmico, tendo em vista o cenário da Reforma Psiquiátrica. Ainda que nesse contexto tenha havido o avanço dos serviços substitutivos, especialmente os Centros de Atenção Psicossocial (CAPS), ainda é uma realidade o fato de que "uma grande parte do sofrimento psíquico menos grave continua sendo objeto do trabalho de ambulatórios e da Atenção Básica, em qualquer uma de suas formas". (9, p. 2)

Conforme citado anteriormente, fizeram parte desse processo vinte e um estagiários e a ação executada correspondeu às etapas do plano descrito no item 2 , levando-o ao delineamento do perfil dos cadastrados abordados e acompanhados pelos estagiários, o qual é apresentado no Quadro 1:

\begin{tabular}{|l|l|}
\hline Faixa Etária & $9,1 \%$ (10 a 20 anos; $15,38 \%$ menor de 18 anos); \\
& $36,4 \%$ (21 a 40 anos); \\
& $31,8 \%$ (41 a 60 anos); \\
& $22,7 \%$ (60 anos em diante). \\
\hline Sexo & $50 \%$ (feminino) \\
& $50 \%$ (masculino) \\
\hline \multirow{5}{*}{ Escolaridade } & $43,47 \%$ (Ensino Fundamental; \\
& $30,43 \%$ (Ensino Médio); \\
& $8,6 \%$ (Alfabetização); \\
& $8,6 \%$ (Ensino Superior); \\
& $4,34 \%$ (nunca estudou); \\
& $8,6 \%$ (estuda atualmente). \\
& $18,2 \%$ (04) casados; \\
& $77,3 \%$ (17) solteiros; \\
& $4,5 \%$ (01) viúvo. \\
\hline Estado Civil & $7,60 \%$ têm dificuldades em arranjar emprego \\
& $7,69 \%$ estão aposentadas; \\
& $7,69 \%$ deixaram o trabalho por cansaço \\
& $23,07 \%$ foram demitidos; \\
\hline Trabalho & $23,07 \%$ não tinham motivos especificados \\
& $15,38 \%$ deixaram o emprego devido à saúde \\
& $15,38 \%$ não trabalham, pois, ainda não alcançaram a maioridade. \\
\hline
\end{tabular}


Quadro 1 - Perfil dos 22 (vinte e dois) sujeitos acompanhados pelos estagiários e cadastrados na Unidade de Saúde da Família do Candeal - Distrito Sanitário de Brotas - 2010

Dos 22 cadastrados, identificados para o trabalho, aqui, identificados como sujeitos, 12 deles (54,5\%) eram assistidos pela Equipe A e 10 (45,5\%) pela Equipe B.

$\mathrm{Na}$ construção do roteiro de entrevista que foi aplicado pelos estudantes quando dos encontros com esses cadastrados, foi considerado, naturalmente, além dos dados sobre o seu perfil, aqueles outros referentes ao campo da saúde mental dos sujeitos. Para isso, foram tomados como referência, indicadores elaborados e divulgados em publicações oficiais para a inclusão de ações relativas à saúde mental na Atenção Básica. ${ }^{(9)}$ De acordo com as diretrizes para as políticas públicas em saúde, é necessário consolidar indicadores nessa área, buscando suprir o Sistema de Informações da Atenção Básica (SIAB) dos dados dessa realidade, como uma forma estratégica de apoio para a avaliação e o planejamento em saúde, evitando, com isso, a exclusão dos casos graves de transtorno mental, dos cuidados necessários na atenção primária, reduzindo consequentemente o seu encaminhamento para a institucionalização. ${ }^{(9)}$

Assim, encontra-se em discussão no âmbito do Ministério da Saúde, a incorporação de tais indicadores de monitoramento relacionados à saúde mental naquele sistema - o SIAB ampliando a visibilidade da saúde mental quando dos cuidados com a saúde integral na rede de atenção primária à saúde.

Na prática de estágio foram utilizados nos questionários, os indicadores de risco à saúde mental transcritos no gráfico abaixo, os quais estão acompanhados do resultado quantitativo dos sujeitos. 


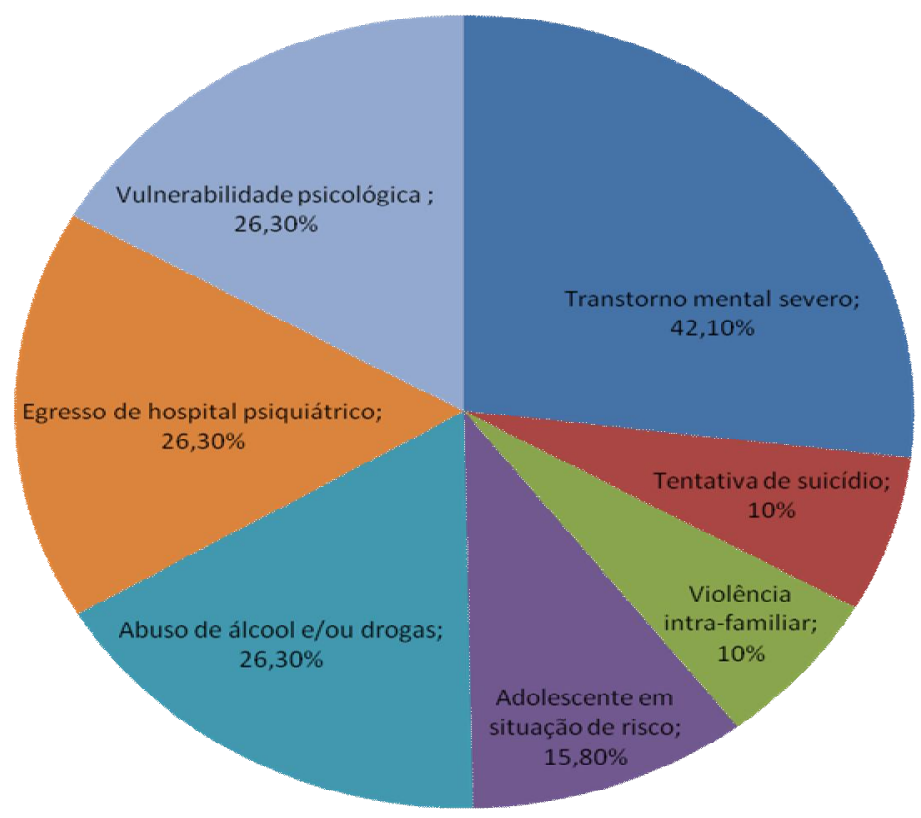

Gráfico 1 - Indicadores de risco - usuários da USF/CANDEAL - 2010

Fonte: Mascarenhas P. Protocolo de acompanhamento em saúde mental no PSF: estágio na Unidade de Saúde da Família. Salvador: EBMSP; 2010. Apresentação PowerPoint.

Uma análise dessas informações coletadas na unidade de saúde da família de referência, demonstra a diversidade dos quadros psicológicos ou psiquiátricos que a ela acessam, vinculando-se aos seus profissionais nas suas demandas em saúde. No modelo da Estratégia de Saúde da Família, a possibilidade de acesso desses profissionais aos contextos familiares dos seus cadastrados, permite ter ela um papel relevante na rede de cuidados. Dados de uma pesquisa do Departamento de Atenção Básica demonstraram que 56\% das equipes de USF preferiram realizar alguma ação de saúde mental na sua rotina de trabalho. A proximidade desses profissionais com as famílias e, em última instância, com a comunidade, coloca-os como "um recurso estratégico para o enfrentamento de agravos vinculados ao uso de álcool, drogas e diversas formas de sofrimento psíquico". (9,p3)

Outros dados relevantes foram coletados, referentes à frequência da intervenção de psicólogos junto aos casos abordados com demonstra o Gráfico 2, a seguir: 


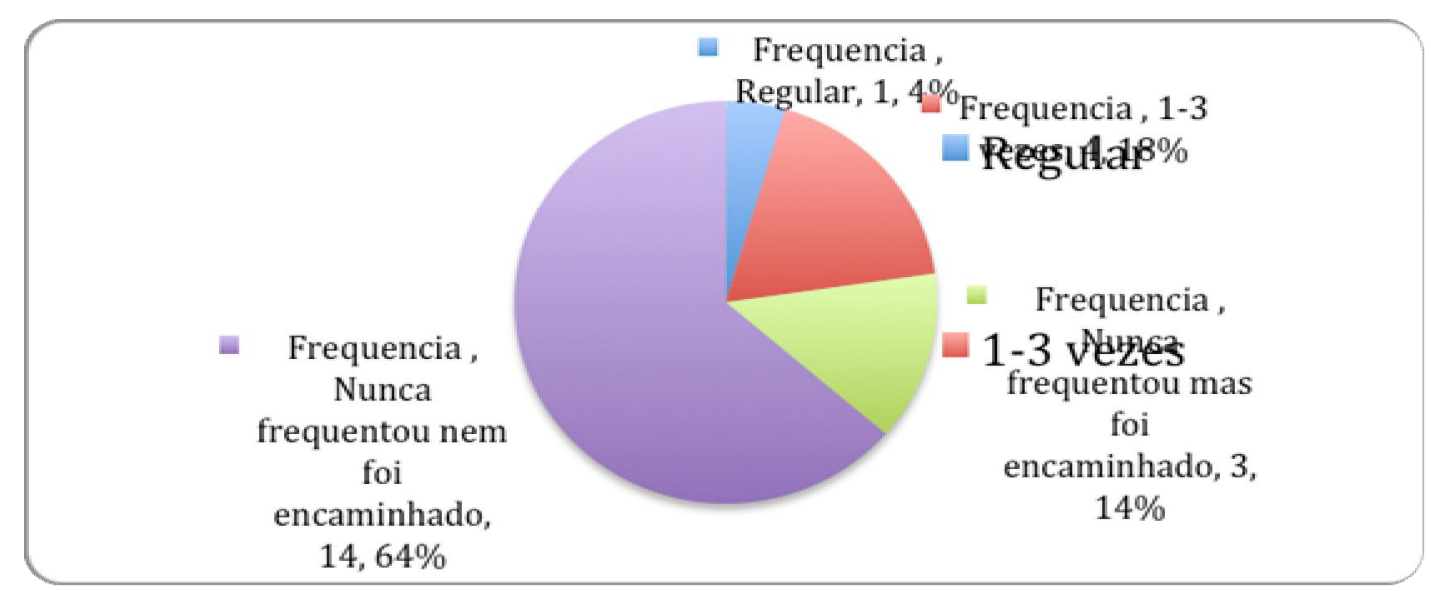

Gráfico 2 - Dados sobre a assistência psicológica aos cadastrados até 2010

Fonte: Mascarenhas P. Protocolo de acompanhamento em saúde mental no PSF: estágio na Unidade de Saúde da Família. Salvador: EBMSP; 2010. Apresentação PowerPoint.

Tendo em vista terem todos eles algum comprometimento ou estarem na área de risco no âmbito da saúde mental, é significativo que apenas 1 cadastrado (4\%) frequenta regularmente e 4 deles (18\%) foi apenas de uma a três vezes em consultas com o psicólogo. A pesquisa indicou que $89 \%$ faz uso de medicamento controlado prescrito por psiquiatra ou pelos profissionais médicos da USF.

A grande maioria - 17 dessas pessoas ou 78\% - enquadram-se naqueles que nunca frequentaram esse tipo de atendimento, mesmo tendo sido encaminhados, como fica evidente. Embora 14 destes tenham dito nunca terem sido encaminhados para o profissional psicólogo, sugerimos uma investigação mais aprofundada para ratificar essa informação a respeito de todos os sujeitos.

Em um levantamento sobre o tipo de apoio necessário, foi citado pelos sujeitos ou pelos seus responsáveis da família, uma diversidade de demandas que podem servir a futuros trabalhos na unidade de saúde de referência, em conjunto com a instituição acadêmica. Segue uma disposição gráfica do que foi levantado: 




Gráfico 3 - Necessidades levantadas junto aos cadastrados ou responsáveis para possíveis intervenções a partir da USF-CANDEAL

Fonte: Mascarenhas P. Protocolo de acompanhamento em saúde mental no PSF: estágio na Unidade de Saúde da Família. Salvador: EBMSP; 2010. Apresentação PowerPoint.

Do que foi citado, muitas das demandas elencadas podem estar atreladas a atividades acadêmicas como práticas ou estágios ligados à educação em saúde ou mesmo intervenções clínicas de caráter individual ou em equipe interdisciplinar.

Dada a natureza das respostas e o que já se tem publicado sobre a temática abordada nessa discussão, podemos inferir ser esta uma realidade entre sujeitos de outros diversos contextos e não apenas desse circunscrito ao Candeal. Isso significa que o incentivo a parcerias entre instituições acadêmicas e unidades básicas de saúde, incluindo as de saúde da família, deve ser fomentado diante do vasto campo de trabalho.

É oportuno discutir a repercussão da ação de estágio nos acadêmicos, visto ter sido esta uma proposta de inovação curricular e, portanto, sem diretrizes anteriores de referência. Para isso, lhes foi entregue um questionário simplificado. Nele, os resultados apontaram para questões ligadas ao aprendizado e à construção de competências e habilidades como futuros profissionais, além de abordar reflexões de ordem subjetiva desses participantes a partir da vivência do processo. Eis alguns resultados.

Aprendi como as relações familiares influenciam o desenvolvimento de algumas doenças. (Aluno 6)

Observei qual a melhor forma de abordar certos assuntos e recolher certas informações. (Aluno 11) 
Foi muito importante conhecer a família no seu contexto, no ambiente domiciliar. Creio que aproxima bastante a relação com o usuário, facilitando o trabalho. (Aluno 14)

Pude ver na prática (de uma USF) como algumas atividades se desenvolvem e os impedimentos encontrados, além de articular a teoria com a prática. (Aluno 16)

Foi muito enriquecedor do ponto de vista acadêmico, conhecer e vivenciar o que foi estudado em saúde coletiva, como também do ponto de vista humano e subjetivo ao ter contato com as pessoas. (Aluno 20)

Face ao exposto, há, pois, que se construir novas tecnologias de serviço, apoiando uma resolutividade significativa no nível da Atenção Primária, no que se refere à promoção e assistência à saúde mental nesse nível de atenção à saúde, anteriormente apenas de responsabilidade dos centros especializados, o que legitima a importância do aprimoramento da prática de estágio apresentada.

Diante da produção de resultados efetivada no nível da comunidade e da formação, sugerimos que a prática de estágio, aqui apresentada, sirva de incentivo a novas iniciativas, atrelando o ensino à prática comunitária.

\section{REFERÊNCIAS}

1. Bastos RMR, França MDA, Duque KCD. Genograma: a utilização deste instrumento gráfico pelas ESF da UBS de Parque Guarani [Internet]. UNIFENAS: Alfenas, 2008. [citado 22 maio 2011]. Disponível em: http://www.unifenas.br/pesquisa/bolsas/ Pet\%20Sa\%FAde/2008/Genograma\%20texto.

2. Silva JA, Dalmaso ASW. O agente comunitário de saúde e suas atribuições: os desafios para os processos de formação de recursos humanos em saúde. Interface Com Saúde Educ. 2002;6:7583.

3. Zarifian P. Objetivo competência: por uma nova lógica. São Paulo: Atlas; 2001.

4. Beneitone P, Esquetini C, González J, Maletá MM, Siufi G, Wagenaar R. Reflexiones y perspectivas de la educación superior en América Latina. Informe final Proyecto Tuning América Latina 2004-2007 [Internet]. Bilbao: Universidad de Deusto, 2007 [citado 25 maio 2011]. Disponível em: http://tuning.unideusto.org/tuningal/index.php?option=com_docman $\&$ task=docclick \&Itemid $=191 \&$ bid $=54 \&$ limitstart $=0 \&$ limit $=5$ 
5. Andrade Cázares RA. El enfoque por competencias en educación. Ide@ s CONCYTEG 2008;3 (39):53-68.

6. Perrenoud P. Construir as competências desde a escola. Porto Alegre: Artmed; 1999.

7. Segnini LRP. Formas diferenciadas de relações empregatícias e qualificações requeridas em um contexto altamente informatizado: análise do sistema financeiro no Brasil: relatório final. In: Centro de Estudos Educação e Sociedade (CEDES). Programa de pesquisa em ciência e tecnologia qualificação e produção n.: 63.95. 0260. 00. Projeto I- Subprojeto 3, Cap 3.3. Qualificação e competência no trabalho no contexto da reestruturação [Internet]. Campinas: Unicamp; [1997] [citado 26 maio 2011]. Disponível em: http://www.cedes.unicamp.br/pesquisa/artigos/LILIANA/cap3.html\#III.III

8. Burnier S. Pedagogia das competências: conteúdos e métodos. Bol Tec SENAC 2001:21:4860.

9. Ministério da Saúde, Secretaria de Atenção à Saúde (BR). Saúde mental e atenção básica: o vínculo e o diálogo necessários [Internet]. Brasília, 2004 [cited 26 maio 2011]. Disponível em: http://portal.saude.gov.br/portal/arquivos/pdf/diretrizes.pdf. 